\title{
Elevated circulating miR-494 in plasma of children with enterovirus 71 induced hand, foot, and mouth disease and its potential diagnostic value
}

\author{
J. LIU, X.-C. LU, W.-D. ZHOU*
}

Received August 26, 2019; revised January 16, 2020; accepted September 14, 2020

\begin{abstract}
Department of Paediatrics, The Affiliated Huaian No. 1 People's Hospital of Nanjing Medical University, Huai'an, Jiangsu 223300, P. R. China
\end{abstract}

\begin{abstract}
Summary. - Hand, foot, and mouth disease (HFMD) is a common pediatric disease, whose outcome depends of the enterovirus genotype infecting the patient. The present study is focused on the potential diagnostic value and the role of circulating microRNA-494 (miR-494) in enterovirus 71-induced more severe form of HFMD. We included 102 children with enterovirus 71 (EV71)-induced HFMD, 42 coxsackievirus A16 (CA16)-induced HFMD and 102 healthy controls. The plasma and serum samples were collected. The expression level of circulating miR-494 was determined by RT-PCR method. Moreover, ROC curve has been drawn to evaluate the sensitivity and specificity of circulating miR-494 for the diagnosis of EV71induced HFMD. Furthermore, the correlation between the circulating miR-494 and the levels of interleukin 6 (IL-6), interleukin 4 (IL-4) and interferon $\gamma($ IFN- $\gamma$ ) in the serum of patients were analyzed. Circulating miR-494 was significantly increased in plasma of children with EV71-induced HFMD compared with the healthy children or CA16-induced HFMD, and level of miR-494 in the EV71 severe group was significantly higher than the EV71 mild group. Moreover, results of ROC analysis suggested that miR-494 is a sensitive biomarker to distinguish EV71 patients from healthy controls and CA16 patients. Furthermore, IL- 6 and IFN- $\gamma$ were elevated in serum of patients with EV71-induced HFMD and the level of circulating miR-494 in patients with EV71-induced HFMD was positively correlated with the serum levels of both IL-6 and IFN- $\gamma$, respectively. Circulating miR-494 was abnormally up-regulated in plasma of the children with EV71-induced HFMD, and miR-494 may serve as potential biomarker for the diagnosis and treatment of the disease.
\end{abstract}

Keywords: miR-494; HFMD; biomarker; enterovirus 71; inflammation

\section{Introduction}

Hand, foot, and mouth disease (HFMD) is one of the most commonly diagnosed, virus-induced pediatric diseases that may affect millions of children every year (Ventarola et al., 2015; Cox et al.,2018). It has been reported

*Corresponding author. E-mail: drliujuan2019@outlook.com; phone: +86-0517-80872262.

Abbreviations: $\mathrm{CA16}=$ coxsackievirus A16; EV71 = enterovirus 71; HFMD = hand, foot, and mouth disease; IFN- $\gamma=$ interferon $\gamma$; IL-4 = interleukin 4; IL-6 = interleukin 6; miR-494 = microRNA-494; ROC = receiver-operating characteristic curve; $\mathrm{Th}=\mathrm{T}$ helper cells in previous study that more than $70 \%$ cases of HFMD were induced by the enterovirus 71(EV71) or coxsackievirus A16 (CA16) virus (Chen et al., 2013). Usually, HFMD is considered as a self-resolving disease; however, in some of the cases, the rapid spread of CA16 or EV71 virus, especially EV71, can cause severe complications in the nervous system, for example encephalitis and acute flaccid paralysis (Chen et al.,2013; Aswathyraj et al.,2016). The incidence of those severe complications may lead to morbidity and unfavorable clinical outcomes of the patients (Aswathyraj et al., 2016; Lee et al., 2016). Therefore, the accurate and early diagnosis of the disease (especially EV71-induced HFMD) is of great importance for the doctors to make rapid clinical decisions and improve the prognosis of the disease. 
MicroRNAs (miRNAs) are a group of non-coding RNAs, and studies on the roles of miRNAs in different diseases has become an area of interest in recent years. miRNAs can bind to the 3 '-untranslated regions of their target $\mathrm{mR}$ NAs, and then either inhibit the translation of the target mRNAs to the proteins or directly cut the target mRNA to inhibit the expression of their target genes (Wu et al., 2014; Tafrihi et al.,2019). Interestingly, miRNAs can be secreted by cells and those miRNAs can stay stable in peripheral blood. Therefore, the changes of levels of miRNAs in the peripheral blood (so-called circulating miRNA) of patients with different disease may indicate an abnormality in the organs or systems of the patients (Keller et al., 2016).

The diagnostic value of circulating miRNAs in HFMD has also been reported previously (Cui et al., 2011; Min et al., 2018). However, whether circulating microRNA-494 (miR-494) can serve as a diagnostic biomarker for HFMD still requires further investigation. Therefore, in this study, we will explore the diagnostic value of circulating miR-494 for CA16- or EV71-induced HFMD by comparing the expression of miR-494 in plasma of HFDM patients and healthy controls, and also, the correlation between circulating miR-494 and inflammatory cytokines in serum of the patients will also be evaluated.

\section{Material and Methods}

Clinical samples. A total of 154 cases of children with HFMD at the Affiliated Huaian No. 1 People's Hospital of Nanjing Medical University between Jan.2018 and Dec.2018 and 102 healthy volunteers were included in the present study. Of the 154 patients, 102 were infected with EV 71 and 42 were infected with CA16. The diagnosis of HFMD was based on the guideline of hand foot and mouth disease prevention control guide (2008 edition) that was issued by the ministry of health of China(http://www.moh.gov.cn/publicfiles/business/htmlfiles/ mohbgt/s9511/200805/34775.htm). The 102 patients with EV 71-induced HFMD were divided into the mild group $(\mathrm{n}=80)$ and severe group $(n=22)$ based on the clinical symptoms. The baseline information (age, gender) of the children showed no significant differences between the three groups (Table 1). The plasma and serum samples of the patients and healthy controls

Table 1. Baseline information of the HFMD patients and healthy volunteers

\begin{tabular}{lcccc}
\hline & $\begin{array}{c}\text { Control } \\
(\mathbf{n = 1 0 2})\end{array}$ & $\begin{array}{c}\text { EV 71 } \\
(\mathbf{n = 1 0 2})\end{array}$ & $\begin{array}{c}\text { CA 16 } \\
(\mathbf{n = 4 2})\end{array}$ & P-value \\
\hline Age (years) & $2.94 \pm 1.52$ & $3.17 \pm 1.45$ & $3.02 \pm 1.19$ & 0.4974 \\
Gender & & & & 0.8703 \\
Male & 53 & 51 & 23 & \\
Female & 49 & 51 & 19 & \\
\hline
\end{tabular}

were collected and immediately stored at $-80^{\circ} \mathrm{C}$ until further reactions. This study was performed based on the principles of the Helsinki Declaration, and the content of the present study has been reviewed by the ethical committee of the Affiliated Huaian No. 1 People's Hospital of Nanjing Medical University. Both the patients and healthy volunteers have signed the informed consent documents.

Isolation of the plasma total RNAs and real-time quantitative PCR (RT-PCR). The total RNAs in the plasma samples were extracted by the TRIzol ${ }^{\oplus}$ reagent (In vitrogen/Thermofisher Scientific, Waltham, MA, USA) according to the manufacture's protocols, and after extraction, the RNA concentration in each sample was measured by NanoDrop ${ }^{\mathrm{TM}} 2000$ spectrophotometer (Thermofisher Scientific, Waltham, MA, USA). Next, RNAs were reverse transcribed into cDNA by miScript reverse transcription kit (Qigen, Hilden, Germany), and cDNA was then amplified by the Hairpin-it ${ }^{\mathrm{TM}}$ miRNAs qPCR Quantitation Kit (GenePharma, Shanghai, China). ABI 7900 Real-Time PCR System (Applied Biosystems, CA, USA) was used for the PCR reaction. The conditions of the PCR reaction were as follow: $95^{\circ} \mathrm{C}$ for $30 \mathrm{~s}, 40$ cycles of $95^{\circ} \mathrm{C}$ for $5 \mathrm{~s}$ and $60^{\circ} \mathrm{C}$ for $30 \mathrm{~s}$. The primers were purchased from Genscript (Nanjing China) and U6 was used as the internal control gene. The expressions of miR-494 was quantified by normalizing the expression of miR-494 to U6 using $2^{-\Delta \Delta \mathrm{Ct}}$ method.

ELISA. The expression levels of interleukin 4 (IL-4), interferon $\gamma($ IFN- $\gamma$ ) and interleukin 6 (IL-6) in the serum of patients and healthy controls were examined by the commercially available ELISA kits (Nanjing Jiancheng Bioengineering Institute, Nanjing, China).

Statistical analysis. The data are expressed as the mean \pm standard deviation. GraphPad Prism (GraphPad Software, Inc., San Diego, CA) was used for data analysis. The comparison between two groups was analyzed by Student's t-test. The diagnostic accuracy of circulating miR-494 was determined by the receiver operating characteristics (ROC) curve, and Pearson's coefficient was used to analyze the correlation between the circulating miR-494 and inflammatory cytokines. $\mathrm{P}<0.05$ was considered as statistically significant difference.

\section{Results}

Increased expression of circulating miR-494 in plasma of patients with EV71-induced HFMD

First, we performed RT-PCR analysis and compared the levels of circulating miR-494 in the plasma of HFMD children and healthy children. As shown in Fig. 1, we found that the levels of circulating miR-494 were significantly higher in the plasma of patients with EV71-induced HFMD than that of the healthy controls (Fig. 1a, p <0.001) and patients with CA16-induced HFMD (Fig. 1a, p <0.001). Moreover, the expression of miR-494 in the severe disease 
(a)

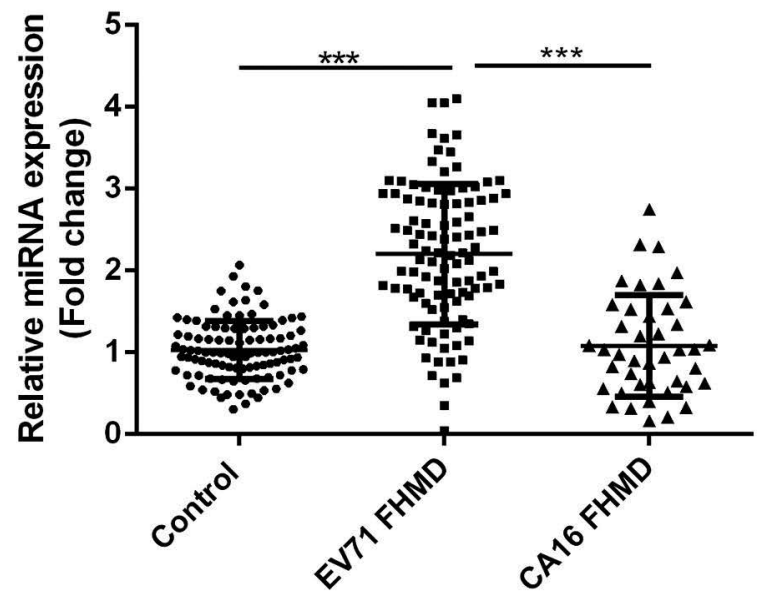

(b)

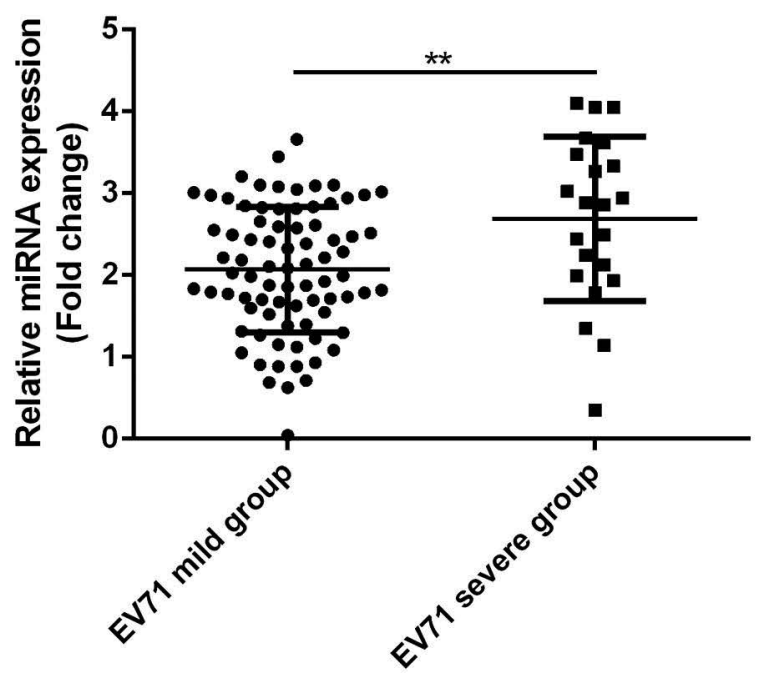

Fig. 1

Comparison of the plasma levels of miR-494 between HFMD and the healthy controls

(a) Comparison between the expression of miR-494 in plasma of healthy control, children with EV71-induced HFMD, and CA-induced HFMD. (b) Comparison between the expression of miR-494 in plasma of the mild group and severe group in children with EV71induced HFMD. ${ }^{* *} \mathrm{p}<0.01,{ }^{* * *} \mathrm{p}<0.001$.

group was significantly higher than in the mild group in the plasma of patients with EV71-induced HFMD (Figure $1 \mathrm{~B}, \mathrm{p}<0.01)$. On the other hand, the plasma level of miR494 showed no significant difference between patients with CA16-induced HFMD and the healthy controls (Fig.1, $\mathrm{p}>0.05)$. (a)

EV71 FHMD vs Control

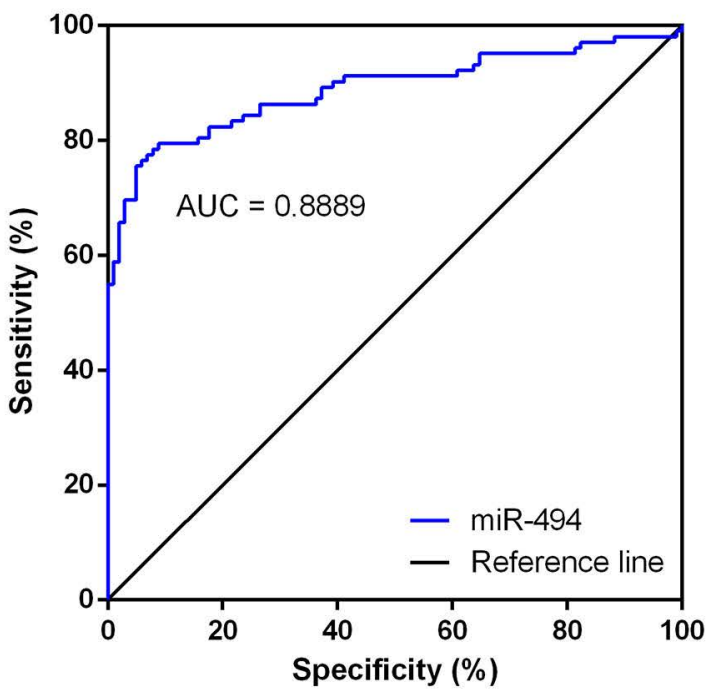

(b)

\section{EV71 FHMD vs CA16 FHMD}

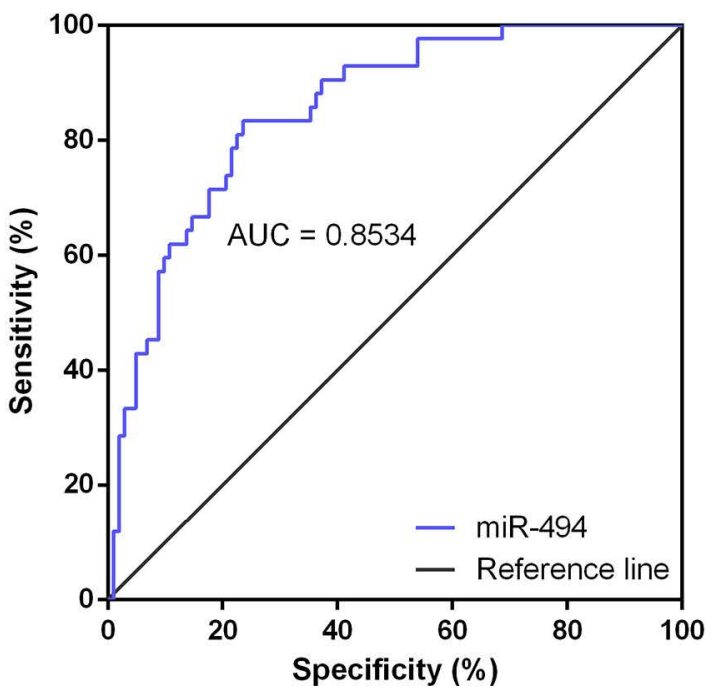

Fig. 2

Circulating miR-494 can serve as a diagnostic marker for EV71induced HFMD

(a) Results of ROC analysis for circulating miR-494 to distinguish children with EV71-induced HFMD and healthy control.(b) Results of ROC analysis for circulating miR-494 to distinguish children with EV71-incude HFMD and CA16-induced HFMD.

Evaluation of the sensitivity and specificity of circulating miR-494 as a diagnostic biomarker for EV71-induced HFMD

The potential diagnostic value of miR-494 for HFMD was examined by drawing the receiver-operating charac- 

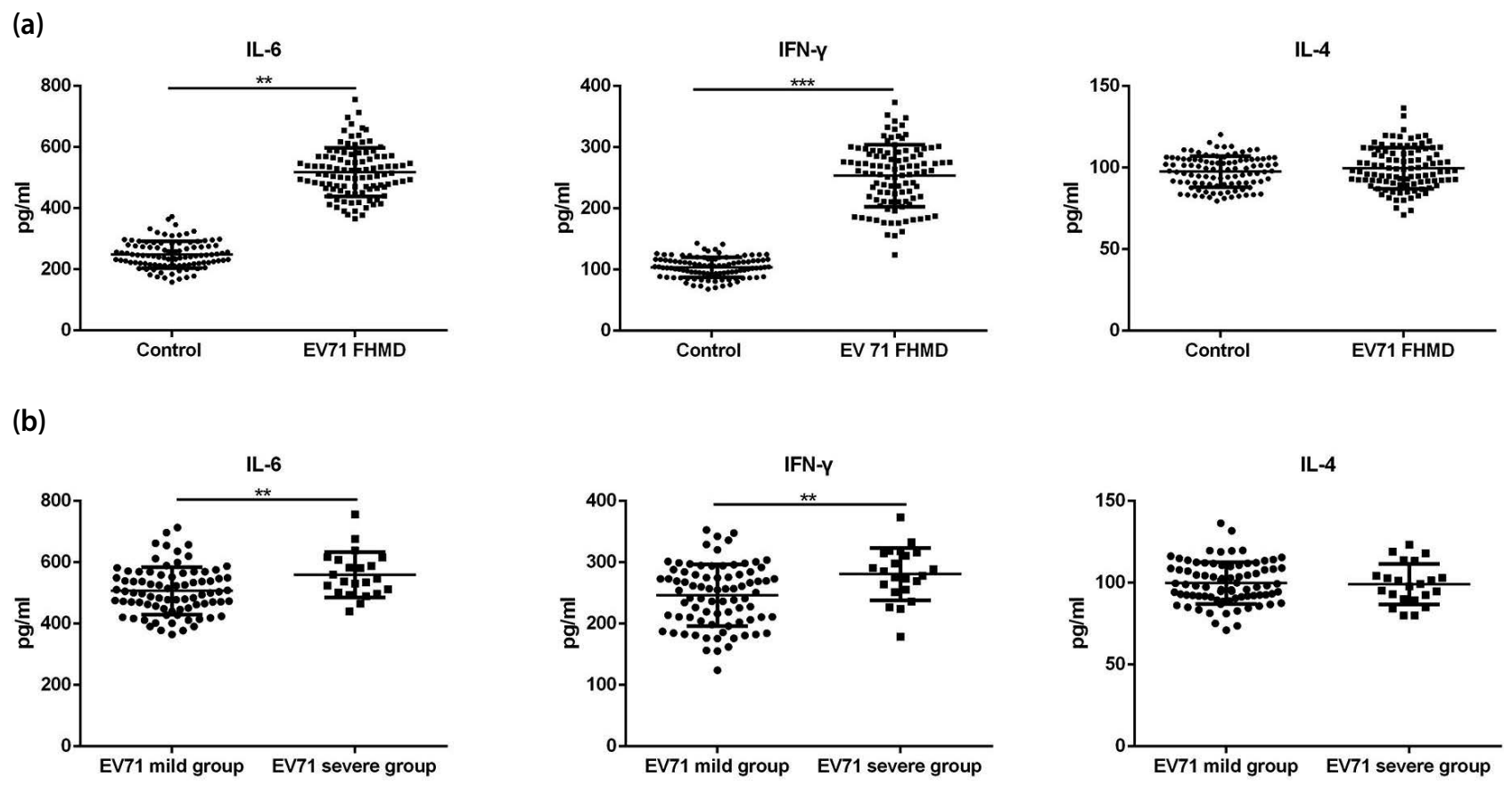

Fig. 3

Elevated inflammatory cytokines in serum of patients with EV71-induced HFMD

(a) Comparison of the serum levels of IFN- $\gamma$, IL-4 and IL-6 between children with EV71-induced HFMD and the healthy controls. (b) Comparison of the serum levels of IFN- $\gamma$, IL- 4 and IL- 6 between mild group and severe group of children with EV71-induced HFMD. ${ }^{* *} \mathrm{p}<0.001$

teristic curve (ROC) curve. We found that the area under curve (AUC) of miR-494 for distinguishing EV71-induced HFMD children and healthy children was 0.8889 (Fig. 2a, $95 \%$ confidence interval (CI), 0.8405 to 0.9372 ), and the AUC for distinguishing EV71-induced HFMD children and CA16-induced HFMD children was 0.8534 (Fig. 2b, 95\% CI, 0.7891 to 0.9177$)$. These results suggested that circulating miR-494 is an accurate biomarker for the diagnosis of EV71- HFMD with high sensitivity and specificity.

Comparison of the serum levels of pro-inflammatory cytokines in serum of children with EV71-induced HFMD

EV71-induced HFMD is known as a viral disease that may lead to inflammatory responses (Ventarola et al., 2015; Cox et al., 2018). Therefore, we compared the expression levels of some pro-inflammatory cytokines in serum of children with EV71-induced HFMD and the healthy children by ELISA method. As shown in Figure, the levels of IFN- $\gamma$ and IL- 6 were significantly increased in serum of children with EV71-induced HFMD compared with healthy children (Fig. 3a, p < 0.01), while on the other hand, the levels of IL-4 showed no significant difference between the two groups (Fig. 3a, p >0.05). Moreover, for the children with EV71-induced HFMD, the levels of both IFN- $\gamma$ and IL- 6 were significantly increased in serum of the severe group compared with the mild group (Fig. $3 \mathrm{~b}$, $\mathrm{p}<0.01$ ), and the level of IL-4 showed no significant difference (Fig. 3b, p >0.05).

Correlation analysis between the circulating $\mathrm{miR}$ 494 and the serum levels of IFN- $\gamma$ and IL-6 in children with EV71-induced HFMD

Finally, we compared the expression levels of the circulating miR-494 and the pro-inflammatory cytokines in serum of children with EV71-induced HFMD. We found the expression level of circulating miR-494 was positively correlated with the serum levels of both IL-6 (Fig. 4, $r=0.4061, p<0.001)$ and IFN- $\gamma($ Fig. $4, r=0.3383, p=0.0005)$ in children with EV71-induced HFMD.

\section{Discussion}

We have explored the potential diagnostic value of miR-494 in HFMD in the present work. The main findings of this work are: first, the circulating miR-494 was abnormally increased in plasma of the children with 
(a)

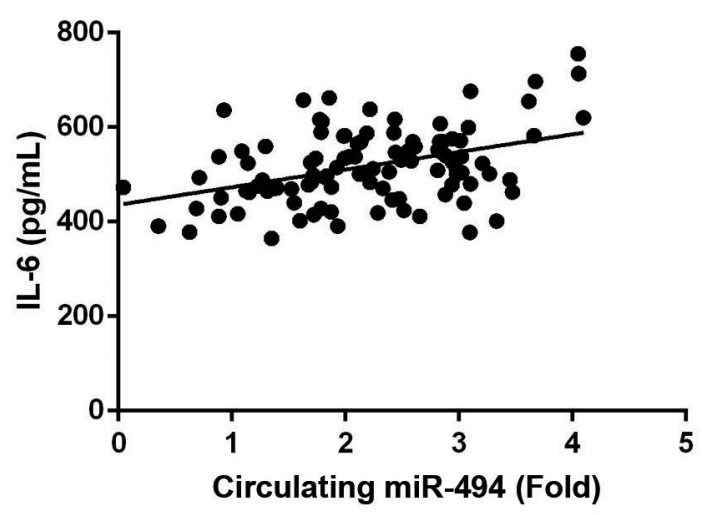

(b)

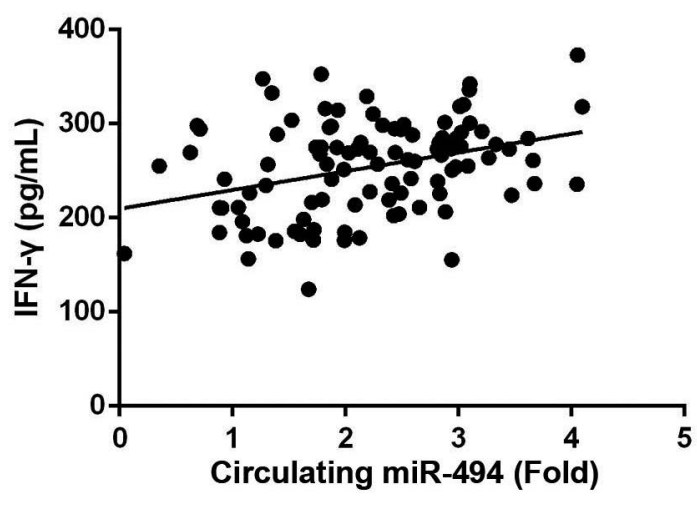

Fig. 4

Correlation between the circulating miR-494 and IFN- $\gamma$ as well as IL-6 in children with EV71-induced HFMD.

EV71-induced HFMD but not CA16-induced HFMD; second, among the patients with EV71-induced HFMD, the circulating miR-494 levels were significantly higher in patients with severe HFMD as compared to mild HFMD; third, the level of the circulating miR-494 was positively correlated with level of IFN- $\gamma$ and IL- 6 in serum of the patients.

In clinical application, EV71 is more likely to induce severe complications than CA16 in patients with HFMD (Cui et al., 2011; Jia et al., 2014). However, the early diagnosis of EV71- and CAV16-induced HFMD is currently difficult. Therefore, to search for specific biomarkers for the early diagnosis of EV71-induced HFMD has great clinical significance. In recent years, great achievements have been made in the field of miRNA related studies. For EV71-induced HFMD, the roles of miRNA in the pathogenesis of the disease have been discussed in many previous studies. For example, Jia et al. have analyzed the expression profile of miRNAs in exosome of patients with HFMD and suggested that exosomal miRNAs may be used for the diagnosis or subtyping of the HFMD infections (Jia et al., 2014). Moreover, Wang et al. suggested that circulating miR-876-5p was elevated in response to EV71 infections (Wang et al.,2016). Feng et al. reported that miR-127-5p can negatively regulate the replication of enterovirus 71 via targeting SCARB2 (Feng et al., 2017).

Results of previous sequencing analysis suggested that miR-494 was one of the most up-regulated miRNAs in response to EV71 infections (Cui et al., 2011; Xun et al., 2015). Therefore, in the present study, we focused on the roles of circulating miR-494 in HFMD and its diagnostic value. As expected, the expression of circulating miR-494 was dramatically up-regulated in plasma of children with EV71-induced HFMD, which was consistent with the results of previous findings (Cui et al., 2011). Interestingly, the circulating miR-494 showed no significant difference between children with CA16-induced HFMD and the healthy control. These results suggested that the overexpression of miR-494 in HFMD was associated with the enterovirus genotype infecting and may be restricted to the EV71 infections. Moreover, the plasma expression of circulating miR-494 was markedly higher in the severe group compared with the mild group of children with EV71-induced HFMD, suggesting that circulating miR-494 may be correlated with the severity of the disease. Furthermore, results of ROC analysis showed that miR-494 can distinguish HFMD children from the healthy control or children with CA16-induced HFMD with high sensitivity and specificity. Taken together, our data suggested that circulating miR-494 was aberrantly up-regulated in EV71-induced HFMD, but not CA16-induced HFMD and may serve as a specific early diagnostic marker of EV71induced HFMD.

HFMD is known as an infectious disease, which may lead to inflammatory responses and to the increased expression of pro-inflammatory cytokines in the peripheral blood (Wang et al., 2014; Lv et al., 2019). IL-6 is known as an activator of the immune system, and the role of IL- 6 in HFMD has been reported previously (Yuan et al., 2015; Lee et al., 2018). On the other hand, IL-4 and IFN- $\gamma$ are the cytokines secreted by the T helper (Th) cells, while IFN- $\gamma$ is known as a marker of Th1 cells, and IL-4 is a marker of Th2 cells (Chen et al., 2014; Das et al., 2014). In our study, we found that IL- 6 and IFN- $\gamma$ were up-regulated in serum of EV71-induced HFMD children, and interestingly, IL-4 showed no significant difference between the two groups. These results suggest that in EV71-induced HFMD, the infection of virus may lead to increased population of Th1 but not Th2 cells and induce the inflammatory response. Moreover, as expected, IFN- $\gamma$ and IL- 6 were markedly increased in serum of the severe group compared with the mild group, and IL-4 showed no significant difference. 
Furthermore, we also found that the level of circulating miR-494 was positively correlated with the levels of IL-6 and IFN- $\gamma$ in patients with EV71-induced HFMD. Based on the results of the present findings, we propose that increased cellular expression of miR-494 may lead to the inhibition of its target genes (which may function as immune suppressors) and induce the inflammatory responses, consequentially increase the secretion of IL-6 and IFN- $\gamma$. However, the related mechanism still needs to be further investigated by cell and animal studies.

In conclusion, the results of the present study suggest that the circulating miR-494 was up-regulated in EV71induced HFMD, and the level of miR-494 was positively associated with the severity of the disease. Our results suggest that miR-494 may function as early diagnostic marker for EV71-induced HFMD.

\section{References}

Aswathyraj S, Arunkumar G, Alidjinou EK, Hober D (2016): Hand, foot and mouth disease (HFMD): emerging epidemiology and the need for a vaccine strategy. Med. Microbiol. Immunol. 205, 397-407. https://doi.org/10.1007/ s00430-016-0465-y

Chen F, Li JJ, Liu T, Wen GQ, Xiang W (2013): Clinical and neuroimaging features of enterovirus71 related acute flaccid paralysis in patients with hand-foot-mouth disease. Asian Pac. J. Trop. Med. 6, 68-72. https://doi. org/10.1016/S1995-7645(12)60203-X

Chen X, Liu H, Peng Y, He L, Zhang Y, Xie Y, Peng X, Liu C, Liu F (2014): Expression and correlation analysis of IL-4, IFN- $\gamma$ and FcaRI in tonsillar mononuclear cells in patients with IgA nephropathy. Cell. Immunol. 289, 70-75. https://doi.org/10.1016/j.cellimm.2014.03.004

Cox B, Levent F (2018): Foot, and Mouth Disease. JAMA 320, 2492. https://doi.org/10.1001/jama.2018.17288

Cui L, Qi Y, Li H, Ge Y, Zhao K, Qi X, Guo X, Shi Z, Zhou M, Zhu B, Guo Y, Li J, Stratton CW, Tang YW, Wang H (2011): Serum microRNA expression profile distinguishes enterovirus 71 and coxsackievirus 16 infections in patients with hand-foot-and-mouth disease. PLoS One 6, e27071. https://doi.org/10.1371/journal.pone.0027071

Das M, Tomar N, Sreenivas V, Gupta N, Goswami R (2014). Effect of vitamin D supplementation on cathelicidin, IFN- $\gamma$, IL-4 and Th1/Th2 transcription factors in young healthy females. Eur. J. Clin. Nutr. 68, 338-343. https:// doi.org/10.1038/ejen.2013.268

Feng C, Fu Y, Chen D, Wang H, Su A, Zhang L, Chang L, Zheng $\mathrm{N}$, Wu Z (2017): miR-127-5p negatively regulates enterovirus 71 replication by directly targeting SCARB2. FEBS Open Bio. 7, 747-758. https://doi.org/10.1002/ $\underline{2211-5463.12197}$
Jia HL, He CH, Wang ZY, Xu YF, Yin GQ, Mao LJ, Liu CW, Deng L (2014): MicroRNA expression profile in exosome discriminates extremely severe infections from mild infections for hand, foot and mouth disease. BMC Infect. Dis. 14, 506. https://doi.org/10.1186/1471-2334-14-506

Keller A, Meese E (2016): Can circulating miRNAs live up to the promise of being minimal invasive biomarkers in clinical settings? Wiley Interdiscip. Rev. RNA 7, 148-756. https://doi.org/10.1002/wrna.1320

Lee KY (2016): Enterovirus 71 infection and neurological complications. Korean J. Pediatr. 59, 395-401. https://doi. org/10.3345/kjp.2016.59.10.395

Lee JY, Son M, Kang JH, Choi UY (2018): Serum interleukin-6 levels as an indicator of aseptic meningitis among children with enterovirus 71-induced hand, foot and mouth disease. Postgrad. Med. 130, 258-263. https:// doi.org/10.1080/00325481.2018.1416257

Lv Y, Wang X. Interleukin-37 Inhibits the Imbalance Between T Helper 17 Cells and Regulatory T Cells in Hand, Foot, and Mouth Disease (2019): J. Interferon Cytokine Res. 39, 421-427. https://doi.org/10.1089/jir.2019.0005

Min N, Sakthi Vale PD, Wong AA, Tan NWH, Chong CY, Chen CJ, Wang RYL, Chu JJH (2018): Circulating Salivary miRNA hsa-miR-221 as Clinically Validated Diagnostic Marker for Hand, Foot, and Mouth Disease in Pediatric Patients. EBioMedicine 31, 299-306. https://doi. org/10.1016/j.ebiom.2018.05.006

Tafrihi M, Hasheminasab E (2019): MiRNAs: Biology, Biogenesis, their Web-based Tools, and Databases. Microrna 8, 4-27. https://doi.org/10.2174/22115366076661808271 $\underline{11633}$

Ventarola D, Bordone L, Silverberg N (2015): Update on handfoot-and-mouth disease. Clin. Dermatol. 33, 340-346. https://doi.org/10.1016/i.clindermatol.2014.12.011

Wang W, Li W, Yang X, Zhang T, Wang Y, Zhong R, Jiao Y, Li T, Jiang T, Tian Y, Wu H (2014): Interleukin-8 is elevated in severe hand, foot, and mouth disease. J. Infect. Dev. Ctries.8, 94-100. https://doi.org/10.3855/jidc.3542

Wang RYL, Weng KF, Huang YC, Chen CJ (2016): Elevated expression of circulating miR876-5p is a specific response to severe EV71 infections. Sci. Rep. 6, 24149. https://doi. org $/ 10.1038 /$ srep24149

Wu Q, Yang Z, Shi Y, Fan D (2014): MiRNAs in human cancers: the diagnostic and therapeutic implications. Curr. Pharm. Des. 20, 5336-5347. https://doi.org/10.2174/1381612820 666140128204914

Xun M, Ma CF, Du QL, Ji YH, Xu JR (2015): Differential expression of miRNAs in enterovirus 71-infected cells. Virol. J.12, 56. https://doi.org/10.1186/s12985-015-0288-2

Yuan A, Li J, Liu P, Chen Z, Hou M, Wang J, Han Z (2015): Association of interleukin-6-572C/G gene polymorphism and serum or cerebrospinal fluid interleukin-6 level with enterovirus 71 encephalitis in Chinese Han patients with hand, foot, and mouth disease. Inflammation 38, 728-735. https://doi.org/10.1007/s10753-014-9983-1 